\title{
High-gradient X-band RF technology for CLIC and beyond
}

\author{
Philip Burrows ${ }^{1}$ \\ Oxford University \\ Oxford, UK \\ E-mail: Philip.Burrowsephysics.ox.ac.uk

\section{Walter Wuensch} \\ CERN \\ Geneva, Switzerland \\ E-mail: Walter.Wuensch@cern.ch

\section{Theodoros Argyropoulos} \\ CERNIIFIC \\ Geneva, Switzerland \Valencia, Spain \\ E-mail: Theodoros. Argyropoulosacern.ch
}

\begin{abstract}
The Compact Linear Collider (CLIC) project is exploring the possibility of constructing a multi$\mathrm{TeV}$ linear electron-positron collider for high-energy frontier physics studies beyond the LHC era. The CLIC concept is based on high-gradient normal-conducting accelerating structures operating at X-band $(12 \mathrm{GHz})$ frequency. We present the status of development, prototyping and testing of structures for operating at gradients of $100 \mathrm{MV} / \mathrm{m}$ and beyond. We report on high-power tests of these structures using the "XBOX" test facilities at CERN and summarize experience with operation at high-gradients. We give an overview of developments for application of the X-band technology to more compact accelerators for use e.g. as X-ray FELs and in medicine.
\end{abstract}

38th International Conference on High Energy Physics

3-10 August 2016

Chicago, USA

\section{${ }^{1}$ Speaker}




\section{Introduction}

The CLIC collaboration has been developing the technology necessary for a multi-TeV range electron-positron collider [1]. A priority of this effort has been to increase the gradient achievable in normal-conducting accelerating structures to above $100 \mathrm{MV} / \mathrm{m}$. Such gradient gives a $3 \mathrm{TeV}$ center of mass energy collider with a total length of $50 \mathrm{~km}$ and allows optimization of gradient for cost and power consumption for lower energies. For example the $380 \mathrm{GeV}$ initial energy stage of CLIC has an optimum gradient of around $70 \mathrm{MV} / \mathrm{m}$ [2].

The underlying strategy has been to take "classical" rf accelerating technology, so that a post-LHC facility can be proposed and built in a timely fashion, understand its fundamental limits, design optimized accelerating structures and demonstrate feasibility with high-power test prototypes. The effort to increase gradient has to take into account the numerous requirements given by the overall design of the CLIC facility. These include that accelerating structures must preserve quality of very low emittance beams, operate with high rf-to-beam power efficiency (high beam-loading), contain features which suppress higher-order-modes (HOMs) and run very stably with a break-down (vacuum arcing) rate of the order of $10^{-7} 1 / \mathrm{m}$ or below [1]. These requirements impose many constraints on the accelerating structures which make the gradient more difficult to achieve.

\subsection{Structure Design and Fabrication}

The high-gradient performance of accelerating structures is highly dependent on the rf design [3]. This dependence also plays a crucial role in the optimization of the whole CLIC facility along with a number of other parameters which are dependent on the rf design such as wakefields, peak power and rf-to-beam efficiency. The performance is also highly dependent on the fabrication technology. The main features of the baseline fabrication technology include:

- Structures are made from micron-precision diamond machined disks, a technology used by both in earlier stages of the CLIC project and the NLC/JLC projects

- The main cells of the structures are bonded at $1040{ }^{\circ} \mathrm{C}$ following the procedure developed in the NLC/JLC project [4].

The baseline technology has been used to produce nearly twenty prototype structures which have run with accelerating gradients in the range of 80 to $120 \mathrm{MV} / \mathrm{m}$, depending on the rf design (see section below).

With the dependence of gradient on rf design now well established, alternative fabrication techniques are currently under investigation. The primary motivation is to reduce the cost of the structure without compromising its performance. The two new main directions are using brazing and constructing the structures from milled halves. In order to investigate the potential of using brazing for high-gradient structures a collaboration with the SwissFEL at PSI was established [5]. Two T24 X-band structures will be built at PSI and will be tested at the CLIC test-stands at CERN following the standardized conditioning protocols. An alternative approach is to construct accelerating structures out of milled halves rather than disks. The major advantage is that the number of parts per structure is significantly reduced, leading to an overall reduction of the total fabrication and handling cost. A prototype accelerating structure T24-open, made in a collaboration between CERN and SLAC, was tested at CERN where an accelerating gradient of $100 \mathrm{MV} / \mathrm{m}$ with a relatively small breakdown rate (BDR) of around $10^{-6} \mathrm{bpp}$ was achieved [6]. 


\subsection{High-gradient testing infrastructure and test results}

In order to test prototype accelerating structures in sufficient numbers, at high gradient, at low BDR and for long periods of time, three X-band, klystron-based test stands have been constructed at CERN. They are collectively known as the "Xboxes". An extensive program in testing CLIC accelerating structures is also being carried out at the NEXTEF facility at KEK. Additional X-band high-gradient testing is carried out at SLAC.

At CERN, XBox-1 and 2 are each equipped with a $50 \mathrm{MW}, 1.5 \mu \mathrm{sec}$ klystron manufactured by CPI, a solid state modulator manufactured by ScandiNova and a SLED-type pulse compressor [7]. Currently each test stand powers a single accelerating structure, which typically requires around $50 \mathrm{MW}$ for $100 \mathrm{MV} / \mathrm{m}$, at a pulse length of about $200 \mathrm{~ns}$ and a repetition rate of $50 \mathrm{~Hz}$. A new test stand, XBox-3, with a significantly different set of parameters is currently being commissioned. It is composed of four power units, each based on a $6 \mathrm{MW}, 5 \mu \mathrm{sec}$ klystron manufactured by Toshiba, a ScandiNova solid-state modulator and a SLED-type pulse compressor. The units are combined in pairs to give the $60 \mathrm{MW}$-range input power needed for testing CLIC prototypes at $100 \mathrm{MV} / \mathrm{m}$. These klystron/modulator units can operate at $400 \mathrm{~Hz}$, but the accelerating structures are limited to around $100 \mathrm{~Hz}$ due to average heating at $100 \mathrm{MV} / \mathrm{m}$ gradient and $200 \mathrm{~ns}$ pulse length. This higher repetition rate can significantly reduce conditioning time since it appears that conditioning progresses with the number of $\mathrm{rf}$ pulses [8].

A summary plot of high-gradient tests carried out on CLIC prototype structures is shown in Fig. 1. The plot shows BDR in units of per pulse per meter of active length vs. the unloaded accelerating gradient. Each square represents the measured performance of a structure. Because testing was made under differing pulse lengths and BDRs (due to practical considerations) the results are scaled, using well established scaling laws, in order to be able to directly compare them. Squares to circles is pulse length scaling and circles to crosses is gradient scaling.

One can distinguish a number of features from the plot. The first is that a gradient above 80 $\mathrm{MV} / \mathrm{m}$ - at very low breakdown rate - has been achieved in a significant number of structures, with the second generation "-24(26)" design consistently achieving over $100 \mathrm{MV} / \mathrm{m} .120 \mathrm{MV} / \mathrm{m}$ has even been achieved for an undamped version of this structure. The results are very significant since they show that a $100 \mathrm{MV} / \mathrm{m}$ gradient for a linear collider is feasible.

Another important insight which has been gained during the high-gradient testing program is the importance of the conditioning process [7]. The effective cost of conditioning structures is potentially high so optimizing the pre-conditioning (i.e. before installation), in-situ conditioning and beam turn-on scenarios is very important. A detailed comparison of the conditioning of different structures has shown that conditioning appears to progress as a function of the number of pulses rather (Fig. 2) than the number of breakdowns [8]. XBox-3 can operate at up to $400 \mathrm{~Hz}$ so the fundamental idea of high-repetition rate conditioning will be tested there.

The results presented above are for structures without beam. In an application such as CLIC, which requires high rf-to-beam efficiency, the fields and power flows inside the structure are modified by the presence of the beam. A dedicated test of the effect on BDR of beam-loading has been carried out using the CTF3 drive beam injector and the XBox-1 power source. Initial results are reported in [9]. 


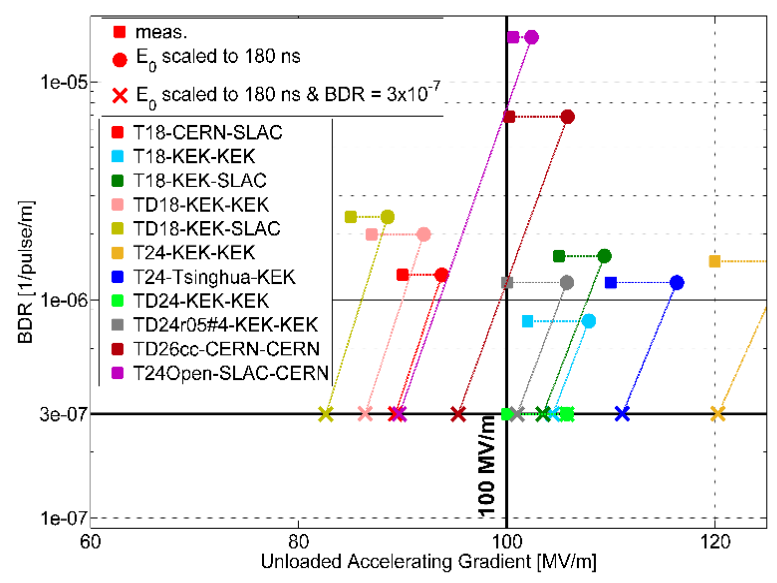

Figure 1: A summary of the high-gradient tests of CLIC prototype structures. All results shown here are without beam loading.

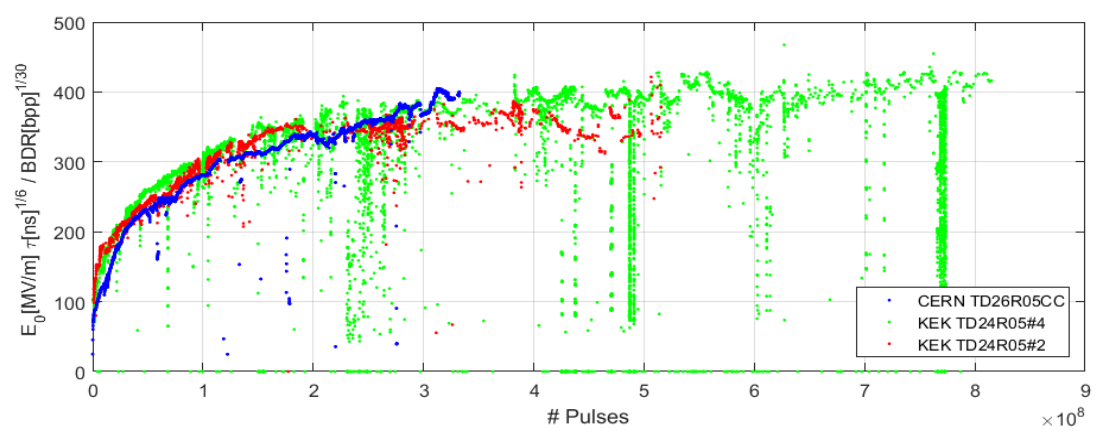

Figure 2: The conditioning histories of three similar rf design accelerating structures tested at KEK and CERN. The gradient is normalized for BDR and pulse length in order to compare test made under different conditions

\subsection{Applications}

The development effort described in this report has been carried out in the context of the CLIC study, which has had the goal of $100 \mathrm{MV} / \mathrm{m}$ acceleration for a TeV-range electron-positron collider. A number of other applications have emerged as potential users of the high-gradient technology developed for CLIC including: XFELs, Compton scattering X-Ray sources and medical linacs for proton and carbon ion therapy. For example, XFELs require electron beam energies in the range up to about $9 \mathrm{GeV}$. Existing normal-conducting XFELs use $\mathrm{S}$ and C-band with gradients below $40 \mathrm{MV} / \mathrm{m}$. The Australian Light Source and SINAP laboratories are considering building XFELs. In both cases, $70 \mathrm{MV} / \mathrm{m}$-range X-band linacs would allow them to obtain their target beam energy and still construct the facilities on their existing sites $[10,11]$. Xband also opens the possibility of repetition rates approaching $1 \mathrm{kHz}$.

Another, very different, area of application of high-gradients is in linac-based proton and carbon ion cancer treatment. Existing facilities use cyclotrons and synchrotrons but linac-based machines have potential advantages in their high repetition rate pulsed time structure (which allows so-called tumour painting) and in smaller, and possibly less expensive, facilities which are easier to integrate in a hospital environment. The CLIC high-gradient team has received funding from the CERN KT fund to construct a high gradient structure for proton acceleration. The structure has been integrated in the TULIP concept developed by the TERA foundation [12]. 


\section{References}

[1] M. Aicheler et. al., CLIC Conceptual Design Report, ANL-HEP-TR-12-51, CERN-2012-005, KEK Report 2012-002, MPP-2012-015.

[2] CLIC and CLICdp collaborations, Updated baseline for a staged Compact Linear Collider, arXiv:1608.07537; CERN-2016-004.

[3] A. Grudiev, S. Calatroni, W. Wuensch, New local field quantity describing the high gradient limit of accelerating structures, Phys. Rev. ST Accel. Beams 12, 102001 (2009).

[4] J. Wang, Research of RF High Gradient Physics and Its Application in Accelerator Structure Development in High Gradient Accelerating Structures, ISBN 978-981-4602-09-9, World Scientific, 2015.

[5] R. Ganter ed., SwissFEL Conceptual Design Report, PSI Beriicht Nr. 10-04, 2012.

[6] W. Wuensch et. al., Fabrication and High-Gradient Testing of an Accelerating Structure Made from Milled Hlaves, LINAC16, East Lansing, MI USA, 2016.

[7] N. Catalan-Lasheras et. al., "Experience Operating an X-band High-Power Test Stand at CERN”, IPAC14, Dresden, Germany, 2014.

[8] A. Degiovanni, W. Wuensch, J. Giner Navarro., Comparison of the conditioning of high gradient accelerating structures, Phys. Rev. Accel. Beams 19, 032001 (2016).

[9] F. Tecker et. al., Beam-Loading Effect on Breakdown Rate in High-Gradient Accelerating Structures, IPAC16, Busan, Korea, 2016.

[10] M. Boland et. al., Plans for an Australian XFEL Using a CLIC X-band Linac, IPAC14, Dresden, Germany, 2014.

[11] W. Fang et. al., $R \& D$; of X-band Accelerating Structure for Compact XFEL at SINAP, LINAC14, Geneva, Switzerland, 2014.

[12] S. Benedetti et. al., Fabrication and testing of a novel S-band backward travelling wave accelerating structure for proton therapy linacs, LINAC16, , East Lansing, MI USA, 2016. 